\title{
Population and prevalence of hypertension in different regions of China
}

\author{
Wang $\mathrm{Jie}^{1,2}$, Yang $\mathrm{Kun}^{2,3^{*}}$, and Dong Wen ${ }^{1,2}$ \\ ${ }^{1}$ School of Information Science and Technology, Yunnan Normal University, Kunming, Yunnan, China \\ ${ }^{2}$ GIS Technology Engineering Research Centre for West-China Resources and Environment of Educational Ministry, Yunnan Normal \\ University, Kunming, Yunnan, China \\ ${ }^{3}$ Faculty Of Geograghy, Yunnan Normal University, Kunming, Yunnan, China
}

\begin{abstract}
Hypertension is the most common chronic disease in the Chinese population. The study analyzes the differences of hypertension in different regions in China from space, analyzes the influencing factors of the disease, and the spatial distribution of different populations. The data comes from CHARLS Harmonize. 22,310 interviewees were selected to respond effectively. Single-factor chi-square test and binary logistic regression were used to analyze the factors affecting hypertension. BMI, urban and rural areas, jogging 1KM, smoking, drink, gender, education status, public medical insurance and hypertension chi-square test are significant, BMI $(\mathrm{OR}=1.19395 \% \mathrm{CI} 1.182 \sim 1.203)$, age $(\mathrm{OR}=1.049,95 \% \mathrm{CI} 1.045 \sim 1.053)$. There is a spatial difference in the prevalence of hypertension in China, which provides a spatial reference for the control and prevention of different regions, so as to better prevent and treat patients with hypertension. Keywords: hypertension, spatial difference, CHARLS data
\end{abstract}

\section{INTRODUCTION}

Cardio-cerebrovascular diseases (CVD) has become a threat to the safety and health of people worldwide. Cardio-cerebrovascular diseases (CVD) accounted for more than one-third of adult death in China in 2010 [1]. Hypertension is one of the most important causes of MORTALITY from CVD. Hypertension can cause serious health problems and increase the risk of death with related diseases [2]. In 2002, 153 million adults suffered from hypertension. In 2013, the control rate of adults with hypertension in China was only $9.7 \%[3,4]$.

About one-third of people in a recent survey have high blood pressure, which has become China's second disease $[5,6]$. With the continuous development of China's economy and the consequent intensification of social aging, the number of people with high blood pressure will continue to rise.

From the distribution of populations in different regions, we studied the spatial distribution of hypertension and found the prevalence in different regions and regions, and spatially discovered hypertension in different regions. The characteristics of the patient population reduce the overall prevalence of hypertension.

\section{Methods}

\subsection{Study population}

CHARLS is a continuous elderly care tracking survey

${ }^{*}$ Corresponding author: janwie@126.com currently carried out in China. The CHARLS Harmonized version is for CHARLS to interact with the global elderly care tracking survey, Select 2015 CHARLS data. CHARLS adopts very strict random sampling. All county-level units in the country are classified by region, According to the PPS (Probability Proportional to Size) method, 150 districts and counties and 450 village-level units are randomly selected in urban and rural and per capita GDP stratification; in each county-level unit, three village-level units are randomly selected according to the PPS method; in each In the village or community, draw residential maps and make a list of households [7-9].

\subsection{Measurements}

Hypertension is defined as self-reported hypertension; the physical test found blood pressure DBP (90mmHg) or SBP $(140 \mathrm{mmHg})$, meeting either of these criteria is considered hypertension [10]. BMI body mass index was used to determine the critical level of overweight based on WGOC using BMI in the general population (24.0), and according to the WS/T 428-2013 (China) standard, determines the optimal BMI Chinese adult critical value is $24 \mathrm{~kg} / x^{2}[11]$. Education level included Less than lower secondary, upper secondary \& vocational training, tertiary.

\subsection{Statistical analysis}

Chi-square for single-factor analysis $x^{2}$ test Hypertension prevalence data, BMI, gender, public health insurance, 
urban and rural areas, education status, jogging for $1 \mathrm{KM}$, smoking and drinking were analyzed to explore the relationship between hypertension and variables, and $p<0.05$ was considered to have significant statistical difference [12]. Binary logistic further scores on age, smoking, drinking, BMI index to further explore the relationship between a factor and hypertension, using odds ratios (ORs) with 95\% confidence intervals (CIs); two-tailed $\mathrm{P}<0.05$ were considered statistically significant [13].

\section{Results}

A total of 25,504 people were surveyed and 22,310 people were selected to be included in the analysis model. Chi-square test showed that BMI, urban and rural area, jogging for $1 \mathrm{KM}$, smoking and drinking were significant $p<0.0001$, gender, education status and public health insurance were $p<0.005$, and the single factor included in the analysis had significant influence on hypertension. BMI (TABLE 1). The prevalence rate gap between male and female was $1.91 \%$, and there was a difference between female and male. The prevalence was $23.12 \%$ in 45-49 years old, $45.19 \%$ in over 70 years old, and $22.07 \%$ under 40 and over 70 years old. The difference of $28.72 \%$ between $50-59$ years old and $39.76 \%$ between 60-69 years old is $11.04 \%$. There is a significant gap in prevalence among people over 60 years old. The education level of junior high school and below accounted for $88.88 \%$ of the interviewed population, the most significant difference in the prevalence of education level is $4.58 \%$, and the difference in education level is not much different in the prevalence rate. Among the respondents, there were 14,469 people in rural areas, and rural people accounted for $64.85 \%$ of the respondents. There was not much difference between urban and rural areas in the prevalence rate, and there was a difference of $3.47 \%$ between urban and rural people. $91.57 \%$ of the respondents purchased public health insurance, which has a high coverage rate. The prevalence of obese people is $24.81 \%$ higher than that of lean people, the prevalence of obese people is $18.71 \%$ higher than that of healthy people, this higher the prevalence of people with higher $\mathrm{BMI}$, the prevalence is also affected by the BMI index.

TABLE 1. Basic characteristics of participants $(n=22310)$

\begin{tabular}{|c|c|c|c|c|c|}
\hline Variable & Over all & Hypertension & Prevalence $(\%)$ & $x^{2}$ & $P$ value \\
\hline Gender & & & & 8.869 & $0.003^{*}$ \\
\hline Male & 10836 & 3709 & 34.22 & & \\
\hline Female & 11474 & 4146 & 36.13 & & \\
\hline Age (years) & & & & 526.318 & $<0.001 * *$ \\
\hline $45-49$ & 1998 & 462 & 23.12 & & \\
\hline $50-59$ & 8144 & 2339 & 28.72 & & \\
\hline $60-69$ & 8201 & 3261 & 39.76 & & \\
\hline$\geq 70$ & 3967 & 1793 & 45.19 & & \\
\hline Education & & & & 12.968 & $0.002 *$ \\
\hline $\begin{array}{c}\text { Less than lower } \\
\text { secondary }\end{array}$ & 19831 & 7054 & 35.57 & & \\
\hline upper secondary \& & 2137 & 677 & 31.67 & & \\
\hline $\begin{array}{c}\text { vocational training } \\
\text { tertiary }\end{array}$ & 342 & 124 & 36.25 & & \\
\hline Drink & & & & 50.025 & $<0.001 * *$ \\
\hline Yes & 7830 & 2516 & 32.13 & & \\
\hline No & 14480 & 5339 & 36.87 & & \\
\hline Smoke & & & & 112.627 & $<0.001 * *$ \\
\hline Yes & 6308 & 1880 & 29.80 & & \\
\hline No & 16002 & 5975 & 37.33 & & \\
\hline Jog $1 \mathrm{KM}$ & & & & 473.736 & $<0.001 * *$ \\
\hline Yes & 11755 & 4914 & 41.80 & & \\
\hline No & 10706 & 2941 & 27.86 & & \\
\hline Region & & & & 26.796 & $<0.001 * *$ \\
\hline Urban & 14469 & 4918 & 33.98 & & \\
\hline Rural & 7841 & 2937 & 37.45 & & \\
\hline Public Health & & & & 4.263 & $0.041^{*}$ \\
\hline Insurance & & & & & \\
\hline Yes & 20430 & 7234 & 35.40 & & \\
\hline No & 1880 & 621 & 33.03 & & \\
\hline BMI & & & & 931.286 & $<0.001^{* *}$ \\
\hline$>18.5$ & 1341 & 281 & 20.95 & & \\
\hline $18.5-24$ & 10809 & 2924 & 27.05 & & \\
\hline$>24$ & 10160 & 4650 & 45.76 & & \\
\hline
\end{tabular}

Note: ${ }^{*} P<0.05 ; * * P<0.01$; BMI, body mass index.

Binary Logistic regression took the incidence of hypertension as the dependent variable, and age, alcohol 
consumption, BMI, and Age score as independent variables. The BMI thin and healthy people have a lower risk of disease than those with a high BMI index. For every 1 unit increase in the BMI index, the risk of disease increases by $19.3 \%$; age in fact, the impact on the prevalence rate tends to be smaller at a younger age (TABLE 2).

TABLE 2.BMI, Age binary logistic regression

\begin{tabular}{c|ccccc}
\hline Risk factors & $\beta$ & $x^{2}$ & $p$ value & OR value & $95 \% C I$ \\
\hline Age & 0.048 & 686.044 & $<0.001^{* *}$ & 1.049 & $1.045 \sim 1.053$ \\
BMI & 0.176 & 1479.693 & $<0.001^{* *}$ & 1.193 & $1.182 \sim 1.203$ \\
\hline
\end{tabular}

Note: $* P<0.05 ; * * P<0.01$; OR, odds ratios; CI, confidence interval.

\section{Discussion}

The study found that 10,836 males and 11,474 females were interviewed, with the number of male patients 3,709 and the number of female patients 4,146 . In the prevalence of young women and men, it is found that there is a difference in the prevalence of men and women. Women should reduce the amount of alcohol consumption to reduce prevalence [14].

We found that the older the prevalence rate is higher, the prevalence rate between the population above 60 and the population below 60 is $7.27 \%$. The prevalence of people over 60 is $41.53 \%$, and this data may continue to rise in the future. A study on the control rate of hypertension among people over 60 found that the prevalence rate is very high, people have a high awareness rate and treatment rate, but a low control rate [15]. The middle-aged and elderly population is the main group of patients with hypertension. Age increases $\mathrm{OR}=1.049$. With the increase of age, people should increase their awareness of controlling hypertension, thereby reducing the prevalence of hypertension.

There was a $24.81 \%$ difference between those with a lean BMI and those who were obese. There was an $18.71 \%$ difference between those with a healthy BMI and those who were obese. The higher the BMI $(\mathrm{OR}=1.193)$, the higher the prevalence rate, BMI has an important influence on the prevalence of hypertension. Overweight and obesity are very common in the Chinese population, and there are differences in obesity between different provinces. Reducing obesity is important for reducing disease [16]. The impact of Chinese adult body weight changes on the prevalence has been found to increase BMI has a significant impact on the prevalence, through lifestyle changes that can reduce the prevalence [17]. Obesity will not only increase the prevalence of high blood pressure but also increase the prevalence of other diseases. More exercise to reduce body weight can greatly reduce the risk of disease.

\section{Conclusion}

The reasonable distribution of medical institutions will bring better medical conditions for hypertension as much as possible and will play a positive role in reducing the risk of cardiovascular and CVD caused by hypertension in the future. The prevalence and population in different regions are different, so different regions should adopt different methods to control and treat people with hypertension.

\section{References}

1. Lewington S, Lacey B, Clarke R, Guo Y, Kong XL, Yang L, et al. The Burden of Hypertension and Associated Risk for Cardiovascular Mortality in China. JAMA Intern Med 2016; 176:524-532.

2. Zhou M, Wang H, Zeng X, Yin P, Zhu J, Chen W, et al Mortality, morbidity, and risk factors in China and its provinces, 1990-2017: a systematic analysis for the Global Burden of Disease Study 2017. The Lancet 2019; 394:1145-1158.

3. Li D, Lv J, Liu F, Liu P, Yang X, Feng Y, et al. Hypertension burden and control in mainland China: Analysis of nationwide data 2003-2012. Int J Cardiol 2015; 184:637-644.

4. Meng XJ, Dong GH, Wang D, Liu MM, Lin Q, Tian S, et al. Prevalence, awareness, treatment, control, and risk factors associated with hypertension in urban adults from 33 communities of China: the CHPSNE study. J Hypertens 2011; 29:1303-1310.

5. Guo QH, Zhang YQ, Wang JG. Asian management of hypertension: Current status, home blood pressure, and specific concerns in China. J Clin Hypertens (Greenwich) 2020; 22:475-478.

6. Jiang Y, Xu Y, Bi Y, Wang L, Zhang M, Zhou M, et al. Prevalence and trends in overweight and obesity among Chinese adults in 2004-10: data from three nationwide surveys in China. The Lancet 2015; 386.

7. Li J, Pang H, Sun Z, Zhao L, Bai C. Health status of middle-aged and older cancer survivors: a nationwide cross-sectional study from the China Health and Retirement Longitudinal Study (CHARLS). Annals of Translational Medicine 2020; 8:183-183.

8. Ma X, Piao X, Oshio T. Impact of social participation on health among middle-aged and elderly adults: evidence from longitudinal survey data in China. BMC Public Health 2020; 20.

9. Zhao Y, Hu Y, Smith JP, Strauss J, Yang G. Cohort profile: the China Health and Retirement Longitudinal Study (CHARLS). Int J Epidemiol 2014; 43:61-68.

10. Song H, Feng D, Wang R, Tang S, Ghose B, Li G, et 
al. Urban-rural disparity in the utilization of national community-based hypertension monitoring service-results from the China Health and Retirement Longitudinal Study, 2015. PeerJ 2019; 7:e7842.

11. Shen C, Zhou Z, Lai S, Tao X, Zhao D, Dong W, et al. Urban-rural-specific trend in prevalence of general and central obesity, and association with hypertension in Chinese adults, aged 18-65 years. BMC Public Health 2019; 19:661.

12. Zhang H, Ni J, Yu C, Wu Y, Li J, Liu J, et al. Sex-Based Differences in Diabetes Prevalence and Risk Factors: A Population-Based Cross-Sectional Study Among Low-Income Adults in China. Front Endocrinol (Lausanne) 2019; 10:658.

13. Tang L, Zhao Q, Han W, Li K, Li J. Association of cardiovascular risk factor clustering and prehypertension among adultsResults from the China health and retirement longitudinal study baseline. Clin Exp Hypertens 2020; 42:315-321.

14. Belanger KM, Crislip GR, Gillis EE, Abdelbary M, Musall JB, Mohamed R, et al. Greater T Regulatory Cells in Females Attenuate DOCA-Salt-Induced Increases in Blood Pressure Versus Males. Hypertension 2020; 75:1615-1623.

15. Sheng CS, Liu M, Kang YY, Wei FF, Zhang L, Li GL, et al. Prevalence, awareness, treatment and control of hypertension in elderly Chinese. Hypertens Res 2013; 36:824-828.

16. Zhang L, Wang Z, Wang X, Chen Z, Shao L, Tian Y, et al. Prevalence of overweight and obesity in China: Results from a cross-sectional study of 441 thousand adults, 2012-2015. Obesity Research \& Clinical Practice 2020; 14:119-126.

17. Ren Q, Su C, Wang H, Wang Z, Du W, Zhang B. Change in Body Mass Index and Its Impact on Incidence of Hypertension in 18-65-Year-Old Chinese Adults. Int J Environ Res Public Health 2016; 13. 